A. Pramesh Rao, G. Swarup and Gopal-Krishna, eds.

\title{
Radio Source Evolution Derived from Low Frequency Surveys
}

\author{
C.A. Jackson
}

Research School of Astronomy \& Astrophysics, The Australian National University, Weston Creek, ACT 2611, Australia

\section{J.V. Wall}

Department of Astrophysics, University of Oxford, Nuclear and Astrophysics Laboratory, Keble Road, Oxford OX1 3RH, UK

\begin{abstract}
We find simple parametric models to describe the space density evolution of radio-loud AGN, treating FRI and FRII radio galaxies separately as the two parent populations in our dual-population unified scheme. In this we use low frequency radio data $(\nu<500 \mathrm{MHz})$, where radio samples are unbiased by Doppler beaming. Incorporated into this latest analysis is a new determination of the local radio luminosity function at $1.4 \mathrm{GHz}$ from galaxies common to both the 2dFGRS and NVSS surveys.
\end{abstract}

\section{Introduction}

All galaxies are radio galaxies. 'Normal' galaxies (like our own) emit at radio wavelengths from the remnants of ongoing stellar birth-death cycles. At higher radio powers are the true 'starburst' galaxies, in which much-enhanced stellar activity results in stronger radio emission. At similar radio powers are the Seyfert galaxies with their small-scale radio core-jet structures. At the top end of the scale are the powerful radio galaxies, blazars and quasars (radio-loud AGN) whose radio jet-lobe structures can extend out to Mpc scales.

It is widely believed that these radio-powerful objects are 'unified' to a greater or lesser degree by projection effects, with a central torus together with relativistic beaming responsible for our classification of objects as radio galaxies, quasars or BL Lac objects. This contribution describes the use of low frequency $(\nu<500 \mathrm{MHz})$ data to avoid projection effects and to define the evolution of the underlying 'parent' populations.

\section{Dual-population unification}

At low radio frequencies $(\nu<500 \mathrm{MHz})$ the radio morphologies of the doublelobed extended sources have been classified by Fanaroff \& Riley (1974) who compared the distances between central maxima of the radio structures to overall size. When the regions of maximum brightness are separated by more than 0.5 times the overall source size, the source is classified as an FRII (edge-brightened). 


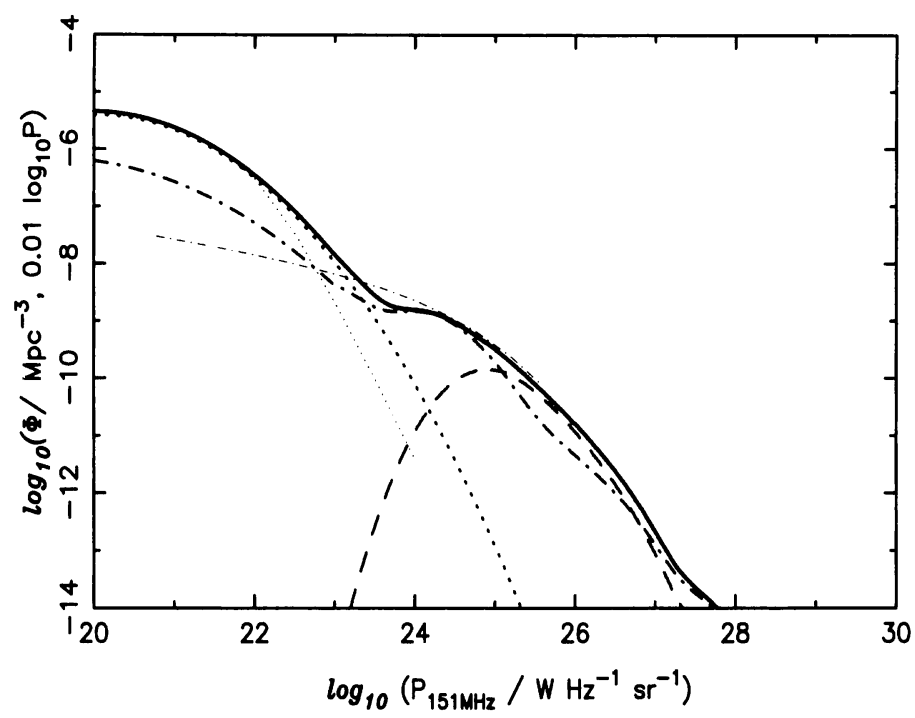

Figure 1. The total LRLF at $151 \mathrm{MHz}$ (solid line) comprises contributions from 3 populations: starburst/Seyfert galaxies (dotted), FRIs (dot-dash) and FRIIs (dashed). For comparison the LRLFs of Condon (1984) are shown as light dots for the starburst/Seyfert galaxies (spirals) and light dot-dash for the FRIs ('monsters').

Where the regions of maximum brightness lies within this limit the source is an FRI (centrally concentrated). The FR-class division correlates with radio power in that the highest radio power objects are predominantly FRIIs whilst those of lower radio power are usually FRIs. The overlap between the classes at middling radio powers can be seen in the radio luminosity function (e.g. Figure 1).

The 'dual-population' unified scheme (Wall \& Jackson, 1997) posits that FRII radio galaxies are the parent population of all quasars and some BL Lac type objects. The FRII-quasar sources are galaxies with class 'A' spectra (Hine \& Longair, 1979), having strong, high-excitation emission lines in their optical/UV spectra. The FRII-BL Lac sources are galaxies with class 'B' spectra (Hine \& Longair, 1979), having only weak, if any, high-excitation lines in their spectra. The second part of the scheme posits that FRI radio galaxies are the parent population of the remainder of the BL Lac type objects.

\section{Methodology}

Our new analysis updates that of Jackson \& Wall (1999) as follows:

(i) The FRI luminosity distribution is defined from the 26 FRIs in 3CRR (Laing, Riley, \& Longair 1983) plus the 9 FRIs in the complete equatorial sample of Best et al. (2000). Radio powers are transposed to $151 \mathrm{MHz}$ using a spectral index $\left(S \propto \nu^{\alpha}\right)$ of -0.75 .

(ii) The low-power $\left(\log _{10} P_{151 \mathrm{MHz}}<23.0 \mathrm{~W} \mathrm{~Hz}^{-1} \mathrm{sr}^{-1}\right)$ local radio luminosity function is calculated from a sample of 927 radio galaxies common to 


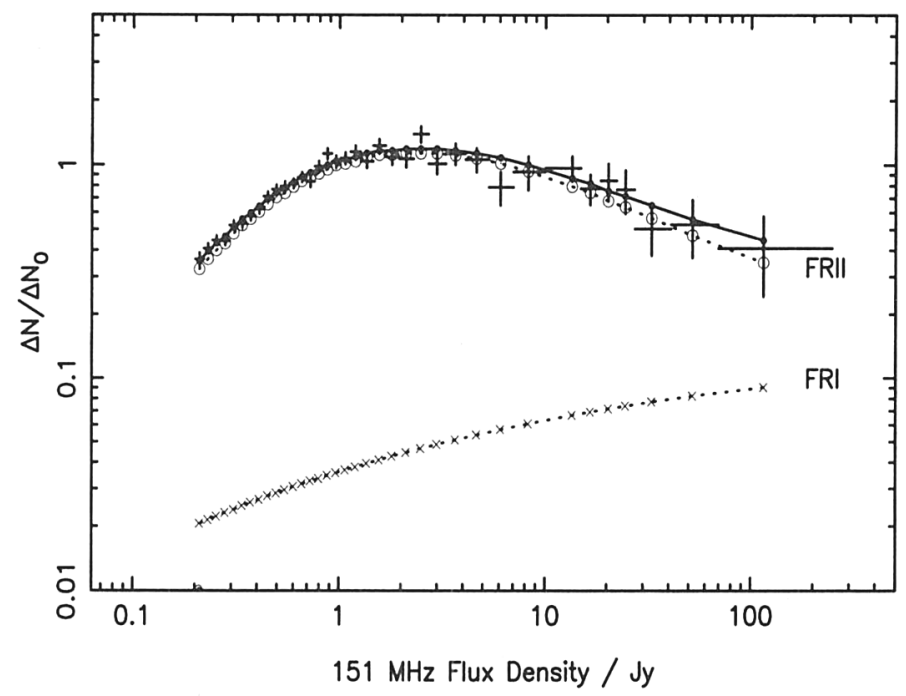

Figure 2. Model (solid line) and observed $(+)$ differential source counts at $151 \mathrm{MHz}$. The contribution from the starburst/Seyfert population falls below the $\Delta N / \Delta N_{0}$ range shown.

both the 2dF Galaxy Redshift survey (2dFGRS, Colless 1999) and the Northern VLA sky survey (NVSS, Condon et al. 1998). We classified the galaxies as starburst, emisssion-line or absorption-line AGN (Sadler et al. 2000). We present the LRLF as the sum of two populations: (i) starburst/Seyfert galaxies and (ii) FRI AGNs. The resultant LRLFs are in reasonable agreement with those of Condon (1984), as shown in Figure 1.

(iii) For the starburst/Seyfert galaxy population we use the evolving luminosity function derived by Haarsma et al (1999). This describes the evolution of the RLF in terms of joint power-law luminosity-density dependence, both having an exponential cut-off at high redshift.

(iv) We use the observed spectral energy distribution of Cygnus A (van Breugel et al. 1999) as a template to calculate the rest-frame radio powers as a function of redshift. This reproduces the observed steepening of $\alpha$ with rest-frame emission frequency.

\section{Results}

We find models of space density evolution which provide successful fits to the differential source count at $151 \mathrm{MHz}$ (comprising 3CRR and 6C surveys (Hales, Baldwin, \& Warner 1988)) for three versions of luminosity dependent density evolution. The contribution from the FRI and FRII populations to the total model source count is shown in Figure 2, where it can be seen that the FRII population dominates. All three models require strong evolution of the FRII population, with only very mild (positive or negative) evolution of the FRI population (Figure 3). The new data together with spectral curvature now permit 

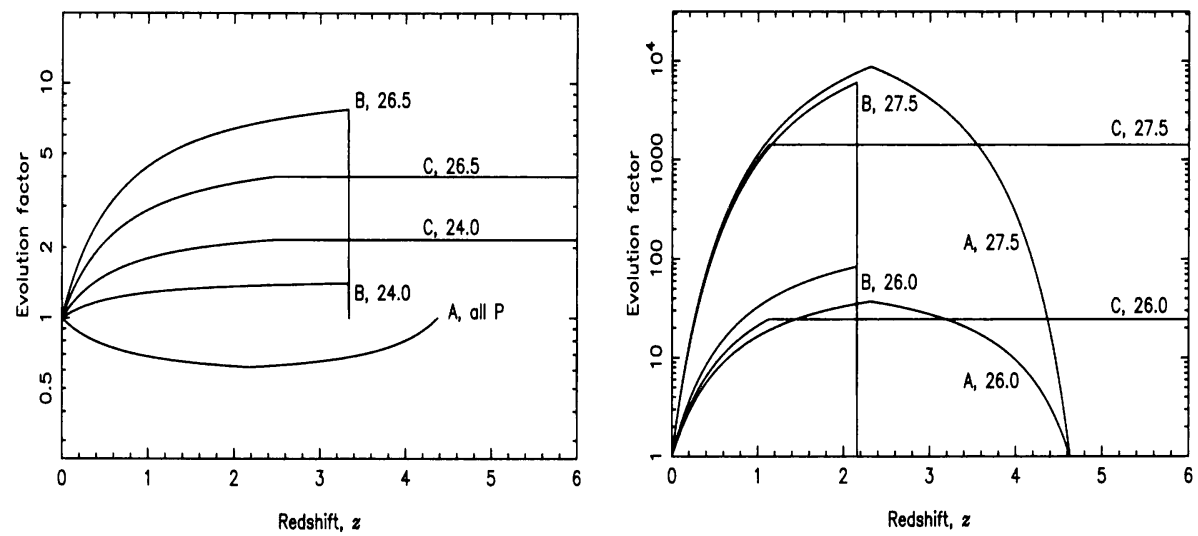

Figure 3. Evolution factors for three models $(\mathrm{A}, \mathrm{B}$ and $\mathrm{C})$ at values for $\log _{10}\left(P_{151 \mathrm{MHz}}\right)$ indicated; FRIs (left) and FRIIs (right).

models which do not require a redshift cutoff. However, strong constraints in this and other regards will be provided by new data sets at low flux densities (e.g. 7C (Rawlings et al., in preparation)), together with revised data for the flat-spectrum quasar sample of Shaver et al. (1996 and in preparation).

\section{References}

Best, P. N., Rottgering, H. J. A., Lehnert, M. D. 2000, MNRAS, in press (astro$\mathrm{ph} / 9903471 \&$ 9912354)

Colless, M. M. 1999, Phil Trans R Soc Lond A, 357, 105

Condon, J. J. 1984, Ap J, 287, 461

Condon, J. J., Cotton, W. D., Greisen, E. W., Yin, Q. F., Perley, R. A., Taylor, G. B., Broderick, J. J. 1998, A J, 115, 1693

Fanaroff, B. L., Riley, J. M. 1974, MNRAS, 167, 31P

Haarsma, D. B., Partridge, R. B., Waddington, I., Windhorst, R. A. 1999, in Proceedings of the 19th Texas Symposium (astro-ph/9904036)

Hales, S. E. G., Baldwin, J. E., Warner, P. J. 1988, MNRAS 234, 919

Hine, R. G., Longair, M. S. 1979, MNRAS, 188, 111

Jackson, C. A., Wall, J. V. 1999, MNRAS, 304, 160

Sadler, E. M., McIntyre, V. J., Jackson, C. A., Cannon, R. D. 2000, PASA, 16, 247

Shaver, P. A., Wall, J. V., Kellermann, K. I., Jackson, C. A., Hawkins, M. R. S. 1996, Nature, 384, 439

van Breugel, W., De Breuck, C., Rottgering, H., Miley, G., Stanford, A. 1999, in Looking Deep in the Southern Sky, ed. R. Morganti, W. Couch, (Heidelberg:Springer-Verlag), 236

Wall, J. V. Jackson, C. A. 1997, MNRAS, 290, L17 